\title{
ESTIMATES FOR DEVIATIONS FROM EXACT SOLUTIONS OF THE CAUCHY PROBLEM FOR MAXWELL'S EQUATIONS
}

\author{
Dirk Pauly, Sergey Repin and Tuomo Rossi \\ Universität Duisburg-Essen, Fakultät für Mathematik \\ Universitätsstr. 2, 45117 Essen, Germany, dirk.pauly@uni-due.de \\ V. A. Steklov Mathematical Institute, St. Petersburg Branch \\ Fontanka 27, 191011 St. Petersburg, Russia; repin@pdmi.ras.ru, and \\ University of Jyväskylä, Department of Mathematical Information Technology \\ P.O. Box 35 (Agora), FI-40014 Jyväskylä, Finland; serepin@jyu.fi \\ University of Jyväskylä, Department of Mathematical Information Technology \\ P.O. Box 35 (Agora), FI-40014 Jyväskylä, Finland; tuomo.rossi@jyu.fi
}

\begin{abstract}
We consider an initial boundary value problem for Maxwell's equations in the space-time cylinder generated by the time interval $[0, T]$. For this hyperbolic type system, we derive guaranteed and computable upper bounds of the difference between the exact solution and any pair of vector fields that belongs to the natural admissible energy class. Our analysis is based upon transformations of the canonical integral relation and Gronwall's inequality and generalizes the method suggested in [22] for the wave equation to the case of the Maxwell's equation.
\end{abstract}

\section{Introduction}

In this paper, we derive computable upper bounds of the distance between the exact solution $(E, H)$ of an initial boundary value problem for the hyperbolic Maxwell system and any pair of vector fields $(\tilde{E}, \tilde{H})$ belonging to the admissible energy class of the problem. As our techniques rely on second order methods and the Maxwell system decouples in its second order version for the electric field $E$ and the magnetic field $H$, we focus on $E$ in our analysis. The vector field $\tilde{E}$ can be considered as an approximation of $E$ computed with the help of a numerical method. In other words, we deduce nonnegative functionals $\mathcal{M}$ (also called error majorants or upper bounds) that depend only on $\tilde{E}$ and known data (coefficients, domain, right hand side and boundary data) and satisfy the following properties:

(1) $\mathcal{E}(E-\tilde{E}) \leq \mathcal{M}(\tilde{E})$ for all admissible $\tilde{E}$;

(2) $\mathcal{M}(\tilde{E})=0$ if and only if $\tilde{E}=E$;

(3) $\mathcal{M}(\tilde{E}) \rightarrow 0$ if $\mathcal{E}(E-\tilde{E}) \rightarrow 0$.

Here, $\mathcal{E}$ is a suitable measure associated with the system (typically it is a $L^{2}$-energy norm defined on the corresponding space-time cylinder $\Omega_{t}$ ).

Functionals $\mathcal{M}$ provide an explicit verification of the accuracy of approximations. The requirements (1)-(3) are quite natural. Indeed, we see that if $\mathcal{M}(\tilde{E})$ is small,

doi:10.5186/aasfm.2011.3641

2010 Mathematics Subject Classification: Primary 65N15, 35L15, 78M30.

Key words: Cauchy problem for Maxwell's equations, bounds of deviations from exact solutions, a posteriori estimates of the functional type. 
then $\tilde{E}$ belongs to a certain neighborhood of the exact solution. Moreover, $\mathcal{M}$ vanishes only at the exact solution $E$. The third property shows that the majorant $\mathcal{M}$ possesses the continuity property with respect to all sequences converging in the topology induced by the energy norm $\mathcal{E}$.

Estimates of such a type (often called functional a posteriori estimates) can be derived by at least two methods. The first method is based on variational techniques and applicable for problems that admit a variational statement. By this method a posteriori error estimates were derived in $[16,17]$ and many other publications (see the book [8] for a systematic overview). Another method is based upon the analysis of the integral identity (variational formulation) that defines the corresponding generalized solution. This method was suggested in [18], where it was also shown that for linear elliptic equations both methods (variational and nonvariational) lead to the same estimates. Later the nonvariational method was also applied to nonlinear elliptic problems and to certain classes of nonlinear problems in continuum mechanics (e.g., for variational inequalities [1, 3, 4, 20]) and to initial boundary value problems associated with parabolic type equations [19]. A consequent exposition of the 'nonvariational' a posteriori error estimation method is presented in the book [21]. Analogous estimates have been recently derived for elliptic problems in exterior domains [13].

In this paper, we are concerned with an initial boundary value problem for Maxwell's equations. For the stationary version of this problem, functional a posteriori estimates have been derived earlier in [14] (the method presented there is applicable for bounded and unbounded domains). However, the hyperbolic Maxwell problem essentially differs from the stationary case and the estimates are derived by a new technique. The derivation method is also based on the analysis of a basic integral relation but uses a rather different modus operandi. The reason for this lies in the specific properties of the respective differential operator involving second order time and spatial derivatives with opposite signs. We overcome the difficulties arising due to this fact with the help of a method suggested in [22] for the wave equation, which is closely related, and deduce computable upper bounds for the distance to the exact solution measured in a canonical $L^{2}$-energy norm.

Our main results are presented in Section 3 by Theorems 2 and 3, which provide computable and guaranteed majorants for the error measures (3.2) and (3.3). These first (and simplest) majorants are derived under stronger assumptions on the regularity of $\tilde{E}$ (which sometimes may be difficult to guarantee in many numerical schemes). In Section 4 we prove corresponding results under weaker assumption on the approximation $\tilde{E}$, which are free of these drawbacks, but have a more complicated structure. Finally, in Section 5 we estimate the error of the approximation of the magnetic field $H$ as well and thus the error of the approximation of the full solution $(E, H)$.

We note that the respective functionals generate new variational problems, where exact lower bounds vanish and are attained only on the exact solution. In applied analysis, the functionals can be used for a posteriori control of errors of approximate solutions obtained by various numerical methods. 


\section{Basic problem}

Let $\Omega$ be an open and connected set, i.e., a domain, in $\mathbf{R}^{3}$ with Lipschitz continuous boundary $\Gamma:=\partial \Omega$ and corresponding outward unit normal vector by $\nu$. Furthermore, let $T>0$ and $I:=(0, T)$. By $\Omega_{t}:=(0, t) \times \Omega$ and $\Gamma_{t}:=(0, t) \times \Gamma$ for all $t>0$ we denote the space-time cylinder and its lateral face, respectively.

We consider the classical initial boundary value problem for Maxwell's equation: Find vector fields $E$ and $H$ (electric and magnetic field), such that

$$
\begin{aligned}
\partial_{t} E-\varepsilon^{-1} \operatorname{curl} H=F & \text { in } \Omega_{T}, \\
\partial_{t} H+\mu^{-1} \operatorname{curl} E=G & \text { in } \Omega_{T}, \\
\nu \times\left. E\right|_{\Gamma}=0 & \text { on } \Gamma_{T}, \\
E(0)=E(0, \cdot)=E_{0} & \text { in } \Omega, \\
H(0)=H(0, \cdot)=H_{0} & \text { in } \Omega .
\end{aligned}
$$

Here $\varepsilon$ and $\mu$ denote time-independent, real, symmetric and positive definite matrices with measurable, bounded coefficients that describe properties of the media (dielectricity and permeability, respectively). For the sake of brevity, matrices (matrixvalued functions) with such properties are called 'admissible'. We note that the corresponding inverse matrices are admissible as well.

We note that $\Omega$ may be bounded or unbounded. Contrary to the stationary cases (i.e., static or time-harmonic equations) the Sobolev spaces used for the solution theory of the Cauchy problem do not differ whether the domain is bounded or not. For instance, in exterior domains one has to work with polynomially weighted Sobolev spaces what naturally would lead to weighted error estimates as well (for a detailed discussion see, e.g., [5, 9, 10, 11, 12]).

By $L^{2}(\Omega)$ we denote the usual scalar $L^{2}$-Hilbert space of square integrable functions on $\Omega$ and by $H^{k}(\Omega), k \in \mathbf{N}$, the usual Sobolev spaces. $H(\Omega)$ denotes the Hilbert space of real-valued $L^{2}$-vector fields, i.e., $L^{2}\left(\Omega, \mathbf{R}^{3}\right)$. In this paper, we restrict our analysis to the case of real-valued functions and vector fields. However, the generalization of our method to complex-valued spaces is straight forward.

We define the spaces

$$
H(\operatorname{curl}, \Omega):=\{\Phi \in H(\Omega) \mid \operatorname{curl} \Phi \in H(\Omega)\}, \quad H\left(\operatorname{curl}^{\circ}, \Omega\right):=\overline{\stackrel{\circ}{C}^{\infty}(\Omega)},
$$

where the closure is taken in the natural graph-norm of $H(\operatorname{curl}, \Omega)$. The homogeneous tangential boundary condition (2.3) is generalized in $H\left(\operatorname{curl}^{\circ}, \Omega\right)$ by Gauß' theorem. Equipped with their natural scalar products all these spaces are Hilbert spaces.

To formulate and obtain a proper Hilbert space solution theory for the latter Cauchy problem, we need some more suitable Hilbert spaces. We set

$$
\mathcal{H}(\Omega):=H(\Omega) \times H(\Omega)
$$

as a set and equip this space with the weighted scalar product

$$
\langle(E, H),(\Phi, \Psi)\rangle_{\mathcal{H}(\Omega)}:=\langle\Lambda(E, H),(\Phi, \Psi)\rangle_{H(\Omega) \times H(\Omega)}=\langle\varepsilon E, \Phi\rangle_{H(\Omega)}+\langle\mu H, \Psi\rangle_{H(\Omega)},
$$

where

$$
\Lambda:=\left[\begin{array}{cc}
\varepsilon & 0 \\
0 & \mu
\end{array}\right]
$$


To simplify the notation, we will write

$$
\|\cdot\|_{\Xi}:=\|\cdot\|_{L^{2}\left(\Xi, \mathbf{R}^{\ell}\right)}, \quad\langle\cdot, \cdot\rangle_{\Xi}:=\langle\cdot, \cdot\rangle_{L^{2}\left(\Xi, \mathbf{R}^{\ell}\right)}, \quad \Xi \subset \mathbf{R}^{N},
$$

and for admissible matrices $A$

$$
\|\cdot\|_{A, \Xi}:=\left\|A^{1 / 2} \cdot\right\|_{\Xi}=\langle A \cdot, \cdot\rangle_{\Xi}^{1 / 2} .
$$

Furthermore, we introduce the linear operator

$$
C_{\Lambda}: \mathcal{D}\left(C_{\Lambda}\right) \subset \mathcal{H}(\Omega) \rightarrow \mathcal{H}(\Omega), \quad(\Phi, \Psi) \mapsto \mathrm{i} \Lambda^{-1} \operatorname{Curl}(\Phi, \Psi),
$$

putting

$$
\mathcal{D}\left(C_{\Lambda}\right):=H\left(\operatorname{curl}^{\circ}, \Omega\right) \times H(\operatorname{curl}, \Omega), \quad \operatorname{Curl}:=\left[\begin{array}{cc}
0 & -\operatorname{curl} \\
\operatorname{curl} & 0
\end{array}\right] .
$$

Then, a solution of (2.1)-(2.5) is to be understood as a solution of the Cauchy problem

$$
\begin{aligned}
\left(\partial_{t}-\mathrm{i} C_{\Lambda}\right)(E, H) & =(F, G), \\
(E, H)(0) & =\left(E_{0}, H_{0}\right) .
\end{aligned}
$$

Utilizing a slight and obvious modification (variation of constant formula) of [7, Theorem 8.5], the Cauchy problem (2.6)-(2.7) has unique solution for all $T$ (we may also replace the interval $I$ by $\mathbf{R}$ ) by spectral theory since $C_{\Lambda}$ is self-adjoint. The spectral theorem suggests

$$
(E, H)(t)=\exp \left(\mathrm{i} t C_{\Lambda}\right)\left(E_{0}, H_{0}\right)+\int_{0}^{t} \exp \left(\mathrm{i}(t-s) C_{\Lambda}\right)(F, G)(s) d s, \quad t \in \bar{I},
$$

as solution. We get:

Theorem 1. Let $(F, G) \in L^{1}(I, \mathcal{H}(\Omega))$ and $\left(E_{0}, H_{0}\right) \in \mathcal{H}(\Omega)$. Then, the Cauchy problem (2.6)-(2.7) is uniquely solvable in

(i) $C^{0}(\bar{I}, \mathcal{H}(\Omega))$;

(ii) $C^{0}\left(\bar{I}, \mathcal{D}\left(C_{\Lambda}\right)\right) \cap C^{1}(\bar{I}, \mathcal{H}(\Omega))$, if additionally $(F, G) \in L^{1}\left(I, \mathcal{D}\left(C_{\Lambda}\right)\right) \cap C^{0}(\bar{I}, \mathcal{H}(\Omega))$ and $\left(E_{0}, H_{0}\right) \in \mathcal{D}\left(C_{\Lambda}\right)$

(iii) $C^{0}\left(\bar{I}, \mathcal{D}\left(C_{\Lambda}^{2}\right)\right) \cap C^{1}\left(\bar{I}, \mathcal{D}\left(C_{\Lambda}\right)\right) \cap C^{2}(\bar{I}, \mathcal{H}(\Omega))$, if additionally $(F, G) \in L^{1}\left(I, \mathcal{D}\left(C_{\Lambda}^{2}\right)\right) \cap C^{0}\left(\bar{I}, \mathcal{D}\left(C_{\Lambda}\right)\right) \cap C^{1}(\bar{I}, \mathcal{H}(\Omega))$ and $\left(E_{0}, H_{0}\right) \in \mathcal{D}\left(C_{\Lambda}^{2}\right)$. to

Here, $(E, H) \in \mathcal{D}\left(C_{\Lambda}^{2}\right)$, if and only if $(E, H)$ and $\left(\varepsilon^{-1} \operatorname{curl} H, \mu^{-1} \operatorname{curl} E\right)$ belong

$$
\mathcal{D}\left(C_{\Lambda}\right)=H\left(\operatorname{curl}^{\circ}, \Omega\right) \times H(\operatorname{curl}, \Omega) .
$$

Remark 1. (i) Theorem 1 holds if we replace the spaces $C^{\ell}$ by spaces of vector fields having such regularity only piecewise, i.e., $C_{p}^{\ell}$, where $\Phi \in C_{p}^{\ell}$, if and only if $\Phi \in C^{\ell-1}$ and $\Phi$ is piecewise $C^{\ell}$.

(ii) To obtain the second order regularity in Theorem 1 (iii) and in view of numerical applications it is sufficient to assume that $\left(E_{0}, H_{0}\right)$ has $H^{2}(\Omega)$-components and that $(F, G)$ and $\varepsilon, \mu$ have $C^{2}\left(\bar{\Omega}_{T}\right)$ - and $C^{1}\left(\bar{\Omega}_{T}\right)$-components with bounded derivatives, respectively. 
If $(E, H)$ admits the second order regularity of Theorem 1 (iii), then we can apply $\partial_{t}+\mathrm{i} C_{\Lambda}$ to $(2.6)$ and obtain

$$
\left(\partial_{t}^{2}+C_{\Lambda}^{2}\right)(E, H)=\Lambda^{-1}(\hat{F}, \hat{G}),
$$

where $(\hat{F}, \hat{G}):=\Lambda\left(\partial_{t}+\mathrm{i} C_{\Lambda}\right)(F, G)$. Equivalently, we have

$$
\left(\partial_{t} \Lambda \partial_{t}-\operatorname{Curl} \Lambda^{-1} \operatorname{Curl}\right)(E, H)=(\hat{F}, \hat{G}) .
$$

Since

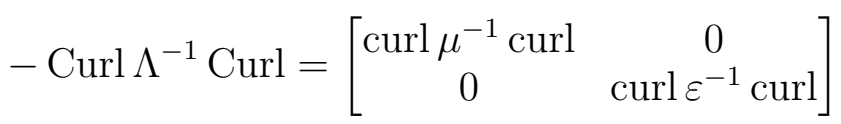

the latter equation decouples for the electric field $E$ and magnetic field $H$. Below we discuss the second order system for the electric field $E$, which reads in classical terms

$$
\begin{aligned}
\left(\partial_{t} \varepsilon \partial_{t}+\operatorname{curl} \mu^{-1} \operatorname{curl}\right) E & =\hat{F} & & \text { in } \Omega_{T}, \\
\nu \times\left. E\right|_{\Gamma} & =0 & & \text { on } \Gamma_{T}, \\
E(0) & =E_{0} & & \text { in } \Omega, \\
\partial_{t} E(0) & =E_{0}^{\prime}:=\varepsilon^{-1} \operatorname{curl} H_{0}+F(0) & & \text { in } \Omega .
\end{aligned}
$$

Henceforth, we assume that for our data $(F, G),\left(E_{0}, H_{0}\right)$ and $\varepsilon, \mu$ the assumptions of Theorem 1 (iii) or Remark 1 (ii) hold, which guarantee the second order regularity of Theorem 1.

Remark 2. Let us impose proper regularity assumptions on the data. We have seen that a solution of the first order problem (2.1)-(2.5) solves also the second order problem (2.8)-(2.11). On the other hand, a solution of the second order problem (2.8)-(2.11) provides also a solution of the original first order problem (2.1)-(2.5). Hence, these two problems are equivalent and uniquely solvable since $(2.1)-(2.5)$ is uniquely solvable. To prove the latter fact it suffices to set

$$
H(t):=\int_{0}^{t}\left(G(s)-\mu^{-1} \operatorname{curl} E(s)\right) d s+H_{0} .
$$

Then, (2.3) and (2.4) hold and (2.2) and (2.5) follow directly. Furthermore, to prove (2.1) we use (2.8) and the above definition of $H$ and obtain

$$
\begin{aligned}
\partial_{t} E(t) & =\int_{0}^{t} \partial_{s}^{2} E(s) d s+E_{0}^{\prime}=-\varepsilon^{-1} \operatorname{curl} \int_{0}^{t} \mu^{-1} \operatorname{curl} E(s) d s+\int_{0}^{t} \varepsilon^{-1} \hat{F}(s) d s+E_{0}^{\prime} \\
& =\varepsilon^{-1} \operatorname{curl} H(t)+\int_{0}^{t}(\underbrace{\left(\varepsilon^{-1} \hat{F}(s)-\varepsilon^{-1} \operatorname{curl} G(s)\right.}_{=\partial_{s} F(s)}) d s+\underbrace{E_{0}^{\prime}-\varepsilon^{-1} \operatorname{curl} H_{0}}_{=F(0)} .
\end{aligned}
$$

\section{First form of the deviation majorant}

Let $\tilde{E}$ be an approximation of $E$. In this section, we assume that

$$
\tilde{E} \in C_{p}^{1}(\bar{I}, H(\operatorname{curl}, \Omega)) \cap C_{p}^{2}(\bar{I}, H(\Omega)) .
$$

Our goal is to find a computable upper bound for the error

$$
e:=E-\tilde{E}
$$


associated with $\tilde{E}$. For all $t \in \bar{I}$ and $\rho \in(0,1)$ we define two nonnegative functions

$$
\begin{aligned}
& n_{\Phi, \rho}(t):=\|\Phi\|_{\varepsilon, \mu^{-1}, \rho, \Omega}^{2}(t):=\left\|\partial_{t} \Phi\right\|_{\varepsilon, \Omega}^{2}(t)+\rho\|\operatorname{curl} \Phi\|_{\mu^{-1}, \Omega}^{2}(t), \\
& N_{\Phi, \rho}(t):=\|\Phi\|_{\varepsilon, \mu^{-1}, \rho, \Omega_{t}}^{2}:=\left\|\partial_{t} \Phi\right\|_{\varepsilon, \Omega_{t}}^{2}+\rho\|\operatorname{curl} \Phi\|_{\mu^{-1}, \Omega_{t}}^{2},
\end{aligned}
$$

which generate natural energy norms for the accuracy evaluation. These functions depend only on $t$. Henceforth, we also use a simplified notation and write $n_{\Phi, \rho}$ instead of $n_{\Phi, \rho}(t)$. In view of Fubini's theorem these quantities are joined by the relations

$$
N_{\Phi, \rho}^{\prime}=n_{\Phi, \rho}, \quad N_{\Phi, \rho}(t)=\int_{0}^{t} n_{\Phi, \rho}(s) d s .
$$

Let $Y$ be a vector field in $C_{p}^{1}(\bar{I}, H(\operatorname{curl}, \Omega))$. We introduce the quantities

$$
\mathcal{F}(\tilde{E}, Y):=\varepsilon \partial_{t}^{2} \tilde{E}+\operatorname{curl} Y-\hat{F}, \quad \mathcal{G}(\tilde{E}, Y):=\mu^{-1} \operatorname{curl} \tilde{E}-Y,
$$

and the weighted sum of their norms

$$
\begin{aligned}
g_{\rho, \gamma}(\tilde{E}, Y ; t):= & \gamma^{-1}\|\mathcal{F}(\tilde{E}, Y)\|_{\varepsilon^{-1}, \Omega_{t}}^{2}+(\gamma \rho)^{-1}\left\|\partial_{t} \mathcal{G}(\tilde{E}, Y)\right\|_{\mu, \Omega_{t}}^{2} \\
& +(1-\rho)^{-1}\|\mathcal{G}(\tilde{E}, Y)\|_{\mu, \Omega}^{2}(t),
\end{aligned}
$$

where $\gamma$ is a positive constant.

Upper bounds of $n_{e, \rho}$ and $N_{e, \rho}$ can be presented in different forms. Below we derive the simplest form of the majorant.

\subsection{Basic form of the error majorant.}

Theorem 2. Let $\tilde{E}$ be an approximation satisfying (3.1) and $\partial_{t} \tilde{E} \in H\left(\operatorname{curl}^{\circ}, \Omega\right)$ for all $t \in I$. Then:

(i) For all $t \in \bar{I}$

$$
n_{e, \rho}(t) \leq \inf _{Y, \gamma} m_{\rho, \gamma}(\tilde{E}, Y ; t), \quad N_{e, \rho}(t) \leq \inf _{Y, \gamma} M_{\rho, \gamma}(\tilde{E}, Y ; t)
$$

where

$$
\begin{aligned}
m_{\rho, \gamma}(\tilde{E}, Y ; t) & :=\gamma \mathrm{e}^{\gamma t} \int_{0}^{t} \mathrm{e}^{-\gamma s} f_{\rho, \gamma}(\tilde{E}, Y ; s) d s+f_{\rho, \gamma}(\tilde{E}, Y ; t), \\
M_{\rho, \gamma}(\tilde{E}, Y ; t) & :=\mathrm{e}^{\gamma t} \int_{0}^{t} \mathrm{e}^{-\gamma s} f_{\rho, \gamma}(\tilde{E}, Y ; s) d s, \\
f_{\rho, \gamma}(\tilde{E}, Y ; t) & :=g_{\rho, \gamma}(\tilde{E}, Y ; t)+z^{+}(e, \tilde{E}, Y ; 0), \\
z^{+}(e, \tilde{E}, Y ; t) & :=n_{e, 1}(t)+2\left|\langle\mathcal{G}(\tilde{E}, Y), \operatorname{curl} e\rangle_{\Omega}\right|(t),
\end{aligned}
$$

and the infima are taken over $\gamma \in \mathbf{R}_{+}$and $Y \in C_{p}^{1}(\bar{I}, H(\operatorname{curl}, \Omega))$.

(ii) If $\tilde{E}(0)=E_{0}$, then for any positive $\gamma$, the majorants $m_{\rho, \gamma}(\tilde{E}, Y ; \cdot)$ and $M_{\rho, \gamma}(\tilde{E}, Y ; \cdot)$ vanish if and only if $\tilde{E}$ coincides with the exact solution $E$ and $Y$ coincides with $\mu^{-1} \operatorname{curl} E$.

Remark 3. (i) We outline that the functionals $f_{\rho, \gamma}(\tilde{E}, Y ; \cdot)$ and $m_{\rho, \gamma}(\tilde{E}, Y ; \cdot)$, $M_{\rho, \gamma}(\tilde{E}, Y ; \cdot)$ depend only on known data, the approximation $\tilde{E}$, the free variable $Y$ and the free parameters $\rho, \gamma$, and do not involve the unknown exact solution $E$. 
(ii) The quantities $\mathcal{F}(\tilde{E}, Y)$ and $\mathcal{G}(\tilde{E}, Y)$ represent errors in two basic relations that jointly form (2.8).

(iii) The term $z^{+}(e, \tilde{E}, Y ; 0)$ represents the error in the initial conditions, i.e., the error at $t=0$. It can be replaced by the weaker (maybe even negative) term $z(e, \tilde{E}, Y ; 0)$, where

$$
\begin{aligned}
z(e, \tilde{E}, Y ; t) & :=n_{e, 1}(t)+2\langle\mathcal{G}(\tilde{E}, Y), \operatorname{curl} e\rangle_{\Omega}(t) \\
& =\left\|\partial_{t} e\right\|_{\varepsilon, \Omega}^{2}(t)+\|\operatorname{curl} e\|_{\mu^{-1}, \Omega}^{2}(t)+2\langle\mathcal{G}(\tilde{E}, Y), \operatorname{curl} e\rangle_{\Omega}(t)
\end{aligned}
$$

But, then Theorem 2 (ii) might not hold. We note that $z^{+}(e, \tilde{E}, Y ; 0)=0$ if and only if $\partial_{t} e(0)=0$ and $\operatorname{curl} e(0)=0$, but $z(e, \tilde{E}, Y ; 0)=0$ if $\partial_{t} e(0)=0$ and $\operatorname{curl} e(0)=0$.

(iv) The free variable $Y$ may be chosen even from the larger space $L^{2}(I, H(\operatorname{curl}, \Omega))$ $\cap C_{p}^{1}(\bar{I}, H(\Omega))$.

All these quantities are explicitly computable once the approximate solution $\tilde{E}$ has been constructed.

Proof of Theorem 2. We start with deriving first order ordinary differential inequalities, which, then lead to the estimates by Gronwall's lemma (see Appendix). Since $\partial_{t} e$ belongs to $H\left(\operatorname{curl}^{\circ}, \Omega\right)$ for all $t \in I$, we have

$$
\begin{aligned}
\partial_{t} n_{e, 1}(t)= & 2\left\langle\varepsilon \partial_{t}^{2} e, \partial_{t} e\right\rangle_{\Omega}(t)+2\left\langle\mu^{-1} \operatorname{curl} e, \operatorname{curl} \partial_{t} e\right\rangle_{\Omega}(t) \\
= & 2\left\langle\hat{F}-\varepsilon \partial_{t}^{2} \tilde{E}, \partial_{t} e\right\rangle_{\Omega}(t)-2\left\langle\mu^{-1} \operatorname{curl} \tilde{E}-Y+Y, \operatorname{curl} \partial_{t} e\right\rangle_{\Omega}(t) \\
= & -2\left\langle\mathcal{F}(\tilde{E}, Y), \partial_{t} e\right\rangle_{\Omega}(t)-2 \partial_{t}\langle\mathcal{G}(\tilde{E}, Y), \operatorname{curl} e\rangle_{\Omega}(t) \\
& +2\left\langle\partial_{t} \mathcal{G}(\tilde{E}, Y), \operatorname{curl} e\right\rangle_{\Omega}(t) .
\end{aligned}
$$

Thus, by integration

$$
\begin{aligned}
n_{e, 1}(t)= & z(e, \tilde{E}, Y ; 0)-2(\underbrace{\langle\mathcal{G}(\tilde{E}, Y), \operatorname{curl} e\rangle_{\Omega}(t)}_{=: S_{1}(t)}-\underbrace{\left\langle\partial_{t} \mathcal{G}(\tilde{E}, Y), \operatorname{curl} e\right\rangle_{\Omega_{t}}}_{=: S_{2}(t)} \\
& +\underbrace{\left\langle\mathcal{F}(\tilde{E}, Y), \partial_{t} e\right\rangle_{\Omega_{t}}}_{=: S_{3}(t)}) .
\end{aligned}
$$

We estimate the terms $S_{1}, S_{2}$ and $S_{3}$ as follows:

$$
\begin{aligned}
& 2\left|S_{1}(t)\right| \leq \alpha\|\operatorname{curl} e\|_{\mu^{-1}, \Omega}^{2}(t)+\alpha^{-1}\|\mathcal{G}(\tilde{E}, Y)\|_{\mu, \Omega}^{2}(t), \\
& 2\left|S_{2}(t)\right| \leq \beta\|\operatorname{curl} e\|_{\mu^{-1, \Omega_{t}}}^{2}+\beta^{-1}\left\|\partial_{t} \mathcal{G}(\tilde{E}, Y)\right\|_{\mu, \Omega_{t}}^{2}, \\
& 2\left|S_{3}(t)\right| \leq \gamma\left\|\partial_{t} e\right\|_{\varepsilon, \Omega_{t}}^{2}+\gamma^{-1}\|\mathcal{F}(\tilde{E}, Y)\|_{\varepsilon^{-1}, \Omega_{t}}^{2} .
\end{aligned}
$$

Here, $\alpha, \beta$ and $\gamma$ are arbitrary nonnegative constants. Let $\alpha:=1-\rho$. Since $\rho \in(0,1)$, we see that $\alpha \in(0,1)$. Next, we set $\beta:=\gamma \rho \in \mathbf{R}_{+}$. Inserting (3.6) into (3.5), we obtain

$$
n_{e, \rho} \leq \gamma N_{e, \rho}+f_{\rho, \gamma}(\tilde{E}, Y ; \cdot)
$$


This relation has two equivalent forms, namely

$$
n_{e, \rho}(t) \leq \gamma \int_{0}^{t} n_{e, \rho}(s) d s+f_{\rho, \gamma}(\tilde{E}, Y ; t)
$$

and

$$
N_{e, \rho}^{\prime}(t) \leq \gamma N_{e, \rho}(t)+f_{\rho, \gamma}(\tilde{E}, Y ; t) .
$$

We apply Gronwall's inequalities, Lemma 1 and 2 of the appendix, and obtain (3.4).

It remains to prove the second part (ii). Substitute $\tilde{E}=E$ and $Y=\mu^{-1} \operatorname{curl} E$ into the majorants. It is trivial that, then

$$
\begin{aligned}
\mathcal{F}(\tilde{E}, Y)=\mathcal{G}(\tilde{E}, Y) & =0, \quad g_{\rho, \gamma}(\tilde{E}, Y ; \cdot)=n_{e, 1}(0)=z^{+}(e, \tilde{E}, Y ; 0)=0, \\
f_{\rho, \gamma}(\tilde{E}, Y ; \cdot) & =0
\end{aligned}
$$

so $m_{\rho, \gamma}(\tilde{E}, Y ; \cdot)=0$ and $M_{\rho, \gamma}(\tilde{E}, Y ; \cdot)=0$. Assume that $m_{\rho, \gamma}(\tilde{E}, Y ; \cdot)=0$ or $M_{\rho, \gamma}(\tilde{E}, Y ; \cdot)=0$. Then,

$$
f_{\rho, \gamma}(\tilde{E}, Y ; \cdot)=g_{\rho, \gamma}(\tilde{E}, Y ; \cdot)=z^{+}(e, \tilde{E}, Y ; 0)=n_{e, 1}(0)=0 .
$$

In particular, $\partial_{t} e=0$, which imlies $e=e(0)=0$. Therefore, $\tilde{E}=E$ and $Y=$ $\mu^{-1} \operatorname{curl} E$ since $g_{\rho, \gamma}(\tilde{E}, Y ; \cdot)=0$ implies $\mathcal{G}(\tilde{E}, Y)=0$.

Remark 4. Theorem 2 implies new variational formulations for the second order problem (2.8)-(2.11). Indeed, for any nonnegative $\gamma$ the functional $m_{\rho, \gamma}(\tilde{E}, Y ; \cdot)$ attains its minimal value (which is equal to zero) if and only if the vector fields $\tilde{E}$ and $\mu Y$ coincide with the exact solution and its curl. All other vector fields (from the above defined functional classes) give larger values to $m_{\rho, \gamma}(\tilde{E}, Y ; \cdot)$. In view of Remark 2, this assertion also holds for the original first order problem (2.1)-(2.5). Of course, these remarks hold also for the functionals $M_{\rho, \gamma}(\tilde{E}, Y ; \cdot)$.

3.2. Refinement of the estimates. We can derive sharper estimates if $\rho$ and $\gamma$ in Theorem 2 are allowed to depend on time, i.e., $\rho: \bar{I} \rightarrow(0,1)$ and $\gamma: \bar{I} \rightarrow \mathbf{R}_{+}$. Then, we replace $n_{\Phi, \rho}(t)$ and $N_{\Phi, \rho}(t)$ by

$$
\begin{aligned}
\tilde{n}_{\Phi, \rho}(t) & :=\left\|\partial_{t} \Phi\right\|_{\varepsilon, \Omega}^{2}(t)+\rho(t)\|\operatorname{curl} \Phi\|_{\mu^{-1}, \Omega}^{2}(t) \\
\tilde{N}_{\Phi, \rho, \gamma}(t) & :=\int_{0}^{t} \gamma(s) \tilde{n}_{\Phi, \rho}(s) d s=\int_{0}^{t} \gamma(s)\left(\left\|\partial_{t} \Phi\right\|_{\varepsilon, \Omega}^{2}(s)+\rho(s)\|\operatorname{curl} \Phi\|_{\mu^{-1}, \Omega}^{2}(s)\right) d s
\end{aligned}
$$

respectively. In this case, $\tilde{N}_{\Phi, \rho, \gamma}^{\prime}=\gamma \tilde{n}_{\Phi, \rho}$ and we modify (3.6) as follows:

$$
\begin{aligned}
& 2\left|S_{1}(t)\right| \leq \alpha(t)\|\operatorname{curl} e\|_{\mu^{-1}, \Omega}^{2}(t)+\alpha^{-1}(t)\|\mathcal{G}(\tilde{E}, Y)\|_{\mu, \Omega}^{2}(t), \\
& 2\left|S_{2}(t)\right| \leq \int_{0}^{t} \beta(s)\|\operatorname{curl} e\|_{\mu^{-1}, \Omega}^{2}(s) d s+\int_{0}^{t} \beta^{-1}(s)\left\|\partial_{t} \mathcal{G}(\tilde{E}, Y)\right\|_{\mu, \Omega}^{2}(s) d s, \\
& 2\left|S_{3}(t)\right| \leq \int_{0}^{t} \gamma(s)\left\|\partial_{t} e\right\|_{\varepsilon, \Omega}^{2}(s) d s+\int_{0}^{t} \gamma^{-1}(s)\|\mathcal{F}(\tilde{E}, Y)\|_{\varepsilon^{-1}, \Omega}^{2}(s) d s .
\end{aligned}
$$

By (3.5) and (3.7) we find that

$$
\begin{aligned}
\tilde{n}_{e, \rho}(t) & \leq \int_{0}^{t} \gamma(s) \tilde{n}_{e, \rho}(s) d s+\tilde{f}_{\rho, \gamma}(\tilde{E}, Y ; t), \\
\tilde{N}_{e, \rho, \gamma}^{\prime}(t) & \leq \gamma(t) \tilde{N}_{e, \rho, \gamma}(t)+\gamma(t) \tilde{f}_{\rho, \gamma}(\tilde{E}, Y ; t),
\end{aligned}
$$


where

$$
\tilde{f}_{\rho, \gamma}(\tilde{E}, Y ; t):=\tilde{g}_{\rho, \gamma}(\tilde{E}, Y ; t)+\left\{\begin{array}{l}
z(e, \tilde{E}, Y ; 0) \\
z^{+}(e, \tilde{E}, Y ; 0)
\end{array}\right. \text { or }
$$

with

$$
\begin{aligned}
\tilde{g}_{\rho, \gamma}(\tilde{E}, Y ; t):= & (1-\rho)^{-1}(t)\|\mathcal{G}(\tilde{E}, Y)\|_{\mu, \Omega}^{2}(t)+\int_{0}^{t} \gamma^{-1}(s)\|\mathcal{F}(\tilde{E}, Y)\|_{\varepsilon^{-1}, \Omega}^{2}(s) d s \\
& +\int_{0}^{t}(\gamma \rho)^{-1}(s)\left\|\partial_{t} \mathcal{G}(\tilde{E}, Y)\right\|_{\mu, \Omega}^{2}(s) d s .
\end{aligned}
$$

We apply Lemma 1 and 2 of the appendix, respectively, and arrive at the following result:

Theorem 3. Let (3.1) hold and $\partial_{t} \tilde{E} \in H\left(\operatorname{curl}^{\circ}, \Omega\right)$ for all $t \in I$. Then, for all $t \in \bar{I}$

$$
\tilde{n}_{e, \rho}(t) \leq \inf _{Y, \gamma} \tilde{m}_{\rho, \gamma}(\tilde{E}, Y ; t), \quad \tilde{N}_{e, \rho, \gamma}(t) \leq \inf _{Y, \gamma} \tilde{M}_{\rho, \gamma}(\tilde{E}, Y ; t)
$$

where

$$
\begin{aligned}
& \tilde{m}_{\rho, \gamma}(\tilde{E}, Y ; t):=\mathrm{e}^{\Gamma(t)} \int_{0}^{t} \mathrm{e}^{-\Gamma(s)} \gamma(s) \tilde{f}_{\rho, \gamma}(\tilde{E}, Y ; s) d s+\tilde{f}_{\rho, \gamma}(\tilde{E}, Y ; t), \\
& \tilde{M}_{\rho, \gamma}(\tilde{E}, Y ; t):=\mathrm{e}^{\Gamma(t)} \int_{0}^{t} \mathrm{e}^{-\Gamma(s)} \gamma(s) \tilde{f}_{\rho, \gamma}(\tilde{E}, Y ; s) d s, \quad \Gamma(t):=\int_{0}^{t} \gamma(s) d s,
\end{aligned}
$$

and the infima are taken over $\gamma: \bar{I} \rightarrow \mathbf{R}_{+}$and $Y \in C_{p}^{1}(\bar{I}, H(\operatorname{curl}, \Omega))$.

Remark 5. It is not difficult to prove that the assertion (ii) of Theorem 2 also holds for the majorants $\tilde{m}$ and $\tilde{M}$ if we use $z^{+}(e, \tilde{E}, Y ; 0)$. If $\gamma$ and $\rho$ are constants, then

$$
\begin{aligned}
& \tilde{m}_{\rho, \gamma}(\tilde{E}, Y ; t)=\gamma \mathrm{e}^{\gamma t} \int_{0}^{t} \mathrm{e}^{-\gamma s} \tilde{f}_{\rho, \gamma}(\tilde{E}, Y ; s) d s+\tilde{f}_{\rho, \gamma}(\tilde{E}, Y ; t), \\
& \tilde{M}_{\rho, \gamma}(\tilde{E}, Y ; t)=\gamma \mathrm{e}^{\gamma t} \int_{0}^{t} \mathrm{e}^{-\gamma s} \tilde{f}_{\rho, \gamma}(\tilde{E}, Y ; s) d s .
\end{aligned}
$$

In this case $\tilde{N}_{e, \rho, \gamma}=\gamma \tilde{N}_{e, \rho, 1}$ and we arrive at the same estimates as in Theorem 2. It is clear that Remarks 3 and 4 hold as well.

\section{Second form of the deviation majorant}

The estimates presented in Theorems 2 and 3 are derived for the approximations $\tilde{E}$ having second order time derivatives. Sometimes, this requirement may be difficult to satisfy. For example standard numerical methods for second order problems often provide approximate solutions having only first order time derivatives (understood in the classical sense). In this section, we derive estimates applicable for approximations of such a type.

As above, $\tilde{E}$ is an approximation of $E$, but now we also introduce a vector field $\tilde{E}_{t}$ considered as an approximation of $\partial_{t} E$. Hence, we define both the error and the error of the time derivative separately by

$$
e:=E-\tilde{E}, \quad e_{t}:=\partial_{t} E-\tilde{E}_{t} .
$$


We note that in general $\tilde{E}_{t} \neq \partial_{t} \tilde{E}$ and therefore $e_{t} \neq \partial_{t} e$. Henceforth, we assume that

$$
e, e_{t} \in C_{p}^{1}(\bar{I}, H(\operatorname{curl}, \Omega)), \quad e_{t} \in H\left(\operatorname{curl}^{\circ}, \Omega\right) \text { for all } t \in I,
$$

which is equivalent to

$$
\tilde{E}, \tilde{E}_{t} \in C_{p}^{1}(\bar{I}, H(\operatorname{curl}, \Omega)), \quad \tilde{E}_{t} \in H\left(\operatorname{curl}^{\circ}, \Omega\right) \text { for all } t \in I .
$$

(We note that the regularity $\tilde{E}_{t} \in C_{p}^{1}(\bar{I}, H(\Omega)) \cap L^{2}(I, H(\operatorname{curl}, \Omega)$ ) would be enough for $\tilde{E}_{t}$.) With two nonnegative, real functions $\rho: \bar{I} \rightarrow(0,1)$ and $\gamma: \bar{I} \rightarrow \mathbf{R}_{+}$we define two energy norms

$$
\begin{aligned}
n_{\Phi, \Psi, \rho}(t) & :=\|\Phi\|_{\varepsilon, \Omega}^{2}(t)+\rho(t)\|\operatorname{curl} \Psi\|_{\mu^{-1}, \Omega}^{2}(t), \\
N_{\Phi, \Psi, \rho, \gamma}(t) & :=\int_{0}^{t} \gamma(s) n_{\Phi, \Psi, \rho}(s) d s=\int_{0}^{t} \gamma(s)\left(\|\Phi\|_{\varepsilon, \Omega}^{2}(s)+\rho(s)\|\Psi\|_{\mu^{-1}, \Omega}^{2}(s)\right) d s .
\end{aligned}
$$

Then, $N_{\Phi, \Psi, \rho, \gamma}^{\prime}=\gamma n_{\Phi, \Psi, \rho}$.

Theorem 4. Assume that (4.1) holds. Then, for all $t \in \bar{I}$

$$
n_{e_{t}, e, \rho}(t) \leq \inf _{Y, \gamma} m_{\rho, \gamma}\left(\tilde{E}, \tilde{E}_{t}, Y ; t\right), \quad N_{e_{t}, e, \rho, \gamma}(t) \leq \inf _{Y, \gamma} M_{\rho, \gamma}\left(\tilde{E}, \tilde{E}_{t}, Y ; t\right),
$$

where

$$
\begin{aligned}
& m_{\rho, \gamma}\left(\tilde{E}, \tilde{E}_{t}, Y ; t\right):=\mathrm{e}^{\Gamma(t)} \int_{0}^{t} \mathrm{e}^{-\Gamma(s)} \gamma(s) f_{\rho, \gamma}\left(\tilde{E}, \tilde{E}_{t}, Y ; s\right) d s+f_{\rho, \gamma}\left(\tilde{E}, \tilde{E}_{t}, Y ; t\right), \\
& M_{\rho, \gamma}\left(\tilde{E}, \tilde{E}_{t}, Y ; t\right):=\mathrm{e}^{\Gamma(t)} \int_{0}^{t} \mathrm{e}^{-\Gamma(s)} \gamma(s) f_{\rho, \gamma}\left(\tilde{E}, \tilde{E}_{t}, Y ; s\right) d s, \quad \Gamma(t):=\int_{0}^{t} \gamma(s) d s,
\end{aligned}
$$

and the infima are taken over $\gamma: \bar{I} \rightarrow \mathbf{R}_{+}$and $Y \in C_{p}^{1}(\bar{I}, H(\operatorname{curl}, \Omega))$. Here,

$$
\begin{aligned}
f_{\rho, \gamma}\left(\tilde{E}, \tilde{E}_{t}, Y ; t\right):= & g_{\rho, \gamma}\left(\tilde{E}, \tilde{E}_{t}, Y ; t\right)+z\left(e, e_{t}, \tilde{E}, Y ; 0\right), \\
g_{\rho, \gamma}\left(\tilde{E}, \tilde{E}_{t}, Y ; t\right):=(1-\rho)^{-1}(t)\|\mathcal{G}(\tilde{E}, Y)\|_{\mu, \Omega}^{2}(t)+2\left\langle\mathcal{G}(\tilde{E}, Y), \operatorname{curl}\left(\tilde{E}_{t}-\partial_{t} \tilde{E}\right)\right\rangle_{\Omega_{t}} & \\
& +\int_{0}^{t} \gamma^{-1}(s)\left\|\hat{\mathcal{F}}\left(\tilde{E}_{t}, Y\right)\right\|_{\varepsilon^{-1}, \Omega}^{2}(s) d s \\
& +\int_{0}^{t}(\gamma \rho)^{-1}(s)\left\|\mu^{-1} \operatorname{curl} \tilde{E}_{t}-\partial_{t} Y\right\|_{\mu, \Omega}^{2}(s) d s, \\
z\left(e, e_{t}, \tilde{E}, Y ; t\right):= & n_{e_{t}, e, 1}(t)+2\langle\mathcal{G}(\tilde{E}, Y), \operatorname{curl} e\rangle_{\Omega}(t),
\end{aligned}
$$

where $\hat{\mathcal{F}}\left(\tilde{E}_{t}, Y\right):=\varepsilon \partial_{t} \tilde{E}_{t}+\operatorname{curl} Y-\hat{F}$.

Remark 6. If $\tilde{E}_{t}=\partial_{t} \tilde{E}$, then the estimates coincide with those of Theorem 3. Furthermore, Remark 3 holds in a similar way. Particularly, $Y$ may be chosen from the larger space

$$
L^{2}(I, H(\operatorname{curl}, \Omega)) \cap C_{p}^{1}(\bar{I}, H(\Omega)) .
$$

If $\gamma>0$ is constant, then

$$
N_{e_{t}, e, \rho, \gamma}(t)=\gamma N_{e_{t}, e, \rho, 1}(t)=\gamma \int_{0}^{t} n_{e_{t}, e, \rho}(s) d s, \quad \Gamma(t)=\gamma t
$$


and the upper bounds simplyfy, i.e.,

$$
\begin{aligned}
& m_{\rho, \gamma}\left(\tilde{E}, \tilde{E}_{t}, Y ; t\right)=\gamma \mathrm{e}^{\gamma t} \int_{0}^{t} \mathrm{e}^{-\gamma s} f_{\rho, \gamma}\left(\tilde{E}, \tilde{E}_{t}, Y ; s\right) d s+f_{\rho, \gamma}\left(\tilde{E}, \tilde{E}_{t}, Y ; t\right), \\
& M_{\rho, \gamma}\left(\tilde{E}, \tilde{E}_{t}, Y ; t\right)=\gamma \mathrm{e}^{\gamma t} \int_{0}^{t} \mathrm{e}^{-\gamma s} f_{\rho, \gamma}\left(\tilde{E}, \tilde{E}_{t}, Y ; s\right) d s .
\end{aligned}
$$

If both $\gamma$ and $\rho$ are constants, then we have

$$
N_{\Phi, \Psi, \rho, \gamma}=\gamma N_{\Phi, \Psi, \rho}, \quad N_{\Phi, \Psi, \rho}:=N_{\Phi, \Psi, \rho, 1} .
$$

In this case,

$$
\begin{aligned}
& n_{\Phi, \Psi, \rho}(t)=\|\Phi\|_{\varepsilon, \Omega}^{2}(t)+\rho\|\operatorname{curl} \Psi\|_{\mu^{-1}, \Omega}^{2}(t), \\
& N_{\Phi, \Psi, \rho}(t)=\|\Phi\|_{\varepsilon, \Omega_{t}}^{2}+\rho\|\operatorname{curl} \Psi\|_{\mu^{-1}, \Omega_{t}}^{2},
\end{aligned}
$$

and

$$
n_{\Phi, \Psi, \rho}=N_{\Phi, \Psi, \rho}^{\prime}, \quad N_{\Phi, \Psi, \rho}(t)=\int_{0}^{t} n_{\Phi, \Psi, \rho}(s) d s .
$$

We arrive at a simplified form of the error majorant.

Theorem 5. Let $\rho \in(0,1)$ and $\tilde{E}$ be an approximation satisfying (4.1). Then, for all $t \in \bar{I}$

$$
n_{e_{t}, e, \rho}(t) \leq \inf _{Y, \gamma} \hat{m}_{\rho, \gamma}\left(\tilde{E}, \tilde{E}_{t}, Y ; t\right), \quad N_{e_{t}, e, \rho}(t) \leq \inf _{Y, \gamma} \hat{M}_{\rho, \gamma}\left(\tilde{E}, \tilde{E}_{t}, Y ; t\right)
$$

where

$$
\begin{aligned}
& \hat{m}_{\rho, \gamma}\left(\tilde{E}, \tilde{E}_{t}, Y ; t\right):=\gamma \mathrm{e}^{\gamma t} \int_{0}^{t} \mathrm{e}^{-\gamma s} f_{\rho, \gamma}\left(\tilde{E}, \tilde{E}_{t}, Y ; s\right) d s+f_{\rho, \gamma}\left(\tilde{E}, \tilde{E}_{t}, Y ; t\right), \\
& \hat{M}_{\rho, \gamma}\left(\tilde{E}, \tilde{E}_{t}, Y ; t\right):=\mathrm{e}^{\gamma t} \int_{0}^{t} \mathrm{e}^{-\gamma s} f_{\rho, \gamma}\left(\tilde{E}, \tilde{E}_{t}, Y ; s\right) d s
\end{aligned}
$$

and the infima are taken over $\gamma \in \mathbf{R}_{+}$and $Y \in C_{p}^{1}(\bar{I}, H(\operatorname{curl}, \Omega))$. Here,

$$
\begin{aligned}
f_{\rho, \gamma}\left(\tilde{E}, \tilde{E}_{t}, Y ; t\right):= & g_{\rho, \gamma}\left(\tilde{E}, \tilde{E}_{t}, Y ; t\right)+z\left(e, e_{t}, \tilde{E}, Y ; 0\right), \\
g_{\rho, \gamma}\left(\tilde{E}, \tilde{E}_{t}, Y ; t\right):= & (1-\rho)^{-1}\|\mathcal{G}(\tilde{E}, Y)\|_{\mu, \Omega}^{2}(t)+2\left\langle\mathcal{G}(\tilde{E}, Y), \operatorname{curl}\left(\tilde{E}_{t}-\partial_{t} \tilde{E}\right)\right\rangle_{\Omega_{t}} \\
& +\gamma^{-1}\left\|\hat{\mathcal{F}}\left(\tilde{E}_{t}, Y\right)\right\|_{\varepsilon^{-1}, \Omega_{t}}^{2}+(\gamma \rho)^{-1}\left\|\mu^{-1} \operatorname{curl} \tilde{E}_{t}-\partial_{t} Y\right\|_{\mu, \Omega_{t}}^{2} .
\end{aligned}
$$

Remark 7. If $\tilde{E}_{t}=\partial_{t} \tilde{E}$, then the estimates coincide with those of Theorem 2 . Again, Remark 3 holds in a similar way. In particular, $Y$ can be chosen from

$$
L^{2}(I, H(\operatorname{curl}, \Omega)) \cap C_{p}^{1}(\bar{I}, H(\Omega)) .
$$

Remark 8. There are different 'zero terms' possible. The (maybe negative) term

$$
z\left(e, e_{t}, \tilde{E}, Y ; 0\right)=n_{e_{t}, e, 1}(0)+2\langle\mathcal{G}(\tilde{E}, Y), \text { curl } e\rangle_{\Omega}
$$

can be replaced by the nonnegative term

$$
\begin{aligned}
z^{+}\left(e, e_{t}, \tilde{E}, Y ; 0\right): & =n_{e_{t}, e, 1}(0)+2\left|\langle\mathcal{G}(\tilde{E}, Y), \operatorname{curl} e\rangle_{\Omega}\right|(0) \\
& =\left\|e_{t}\right\|_{\varepsilon, \Omega}^{2}(0)+\|\operatorname{curl} e\|_{\mu^{-1}, \Omega}^{2}(0)+2 \mid\langle\mathcal{G}(\tilde{E}, Y), \text { curl } e\rangle_{\Omega} \mid
\end{aligned}
$$


or even by

$$
\hat{z}^{+}\left(e, e_{t}, \tilde{E}, Y ; 0\right):=\left\|e_{t}\right\|_{\varepsilon, \Omega}^{2}(0)+2\|\operatorname{curl} e\|_{\mu^{-1}, \Omega}^{2}(0)+\|\mathcal{G}(\tilde{E}, Y)\|_{\mu, \Omega}^{2}(0),
$$

which are easily computable. We have

$$
\left|z\left(e, e_{t}, \tilde{E}, Y ; 0\right)\right| \leq z^{+}\left(e, e_{t}, \tilde{E}, Y ; 0\right) \leq \hat{z}^{+}\left(e, e_{t}, \tilde{E}, Y ; 0\right) .
$$

Similar manipulations can be done with the term $2\left\langle\mathcal{G}(\tilde{E}, Y), \operatorname{curl}\left(\tilde{E}_{t}-\partial_{t} \tilde{E}\right)\right\rangle_{\Omega_{t}}$, taking, e.g., it's absolute value, which leads to some nonnegative $\tilde{g}_{\rho, \gamma}\left(\tilde{E}, \tilde{E}_{t}, Y ; t\right)$. Moreover,

(i) $z\left(e, e_{t}, \tilde{E}, Y ; 0\right)=0$ if $e_{t}(0)=0$ and $\operatorname{curl} e(0)=0$,

(ii) $z^{+}\left(e, e_{t}, \tilde{E}, Y ; 0\right)=0$ if any only if $e_{t}(0)=0$ and $\operatorname{curl} e(0)=0$,

(iii) $\hat{z}^{+}\left(e, e_{t}, \tilde{E}, Y ; 0\right)=0$ if any only if $e_{t}(0)=0$ and curl $e(0)=0$ and $\mu Y(0)=$ curl $\tilde{E}(0)$,

(iv) $\tilde{g}_{\rho, \gamma}\left(\tilde{E}, \tilde{E}_{t}, Y ; \cdot\right)=0$ if and only if $\mu Y=\operatorname{curl} \tilde{E}$ and $\partial_{t} \mu Y=\operatorname{curl} \tilde{E}_{t}$ and $\operatorname{curl} Y=\hat{F}-\varepsilon \partial_{t} \tilde{E}_{t}$.

Therefore, choosing the functional $f_{\rho, \gamma}\left(\tilde{E}, \tilde{E}_{t}, Y ; \cdot\right)$ with the quantities $z^{+}\left(e, e_{t}, \tilde{E}, Y\right.$; $0)$ or $\hat{z}^{+}\left(e, e_{t}, \tilde{E}, Y ; 0\right)$ and $\tilde{g}_{\rho, \gamma}\left(\tilde{E}, \tilde{E}_{t}, Y ; \cdot\right)$ we see that the functional $m_{\rho, \gamma}\left(\tilde{E}, \tilde{E}_{t}, Y ; \cdot\right)$ vanishes, if and only if

$$
\begin{aligned}
\tilde{E}_{t}(0) & =E_{0}^{\prime}, \quad \operatorname{curl} \tilde{E}(0)=\operatorname{curl} E_{0}, \\
\mu Y & =\operatorname{curl} \tilde{E}, \quad \partial_{t} \mu Y=\operatorname{curl} \tilde{E}_{t}, \quad \operatorname{curl} Y=\hat{F}-\varepsilon \partial_{t} \tilde{E}_{t} .
\end{aligned}
$$

Thus, $e_{t}$ and curl $e$ vanish, if and only if $n_{e_{t}, e, \rho}=0$, which is implied by

$$
m_{\rho, \gamma}\left(\tilde{E}, \tilde{E}_{t}, Y ; \cdot\right)=0 .
$$

The latter constraint is equivalent to (4.4) and (4.5). The same holds true for the energy norms $N_{e_{t}, e, \rho}, N_{e_{t}, e, \rho, \gamma}$ and the functionals $M_{\rho, \gamma}\left(\tilde{E}, \tilde{E}_{t}, Y ; \cdot\right), \hat{m}_{\rho, \gamma}\left(\tilde{E}, \tilde{E}_{t}, Y ; \cdot\right)$ and $\hat{M}_{\rho, \gamma}\left(\tilde{E}, \tilde{E}_{t}, Y ; \cdot\right)$.

Proof of Theorem 4. We follow in close lines the proofs of Theorems 2 and 3. Since $e_{t} \in H\left(\operatorname{curl}^{\circ}, \Omega\right)$ and $\partial_{t} e=e_{t}+\tilde{E}_{t}-\partial_{t} \tilde{E}$, we have

$$
\begin{aligned}
& \partial_{t} n_{e_{t}, e, 1}(t)=2\left\langle\varepsilon \partial_{t} e_{t}, e_{t}\right\rangle_{\Omega}(t)+2\left\langle\mu^{-1} \operatorname{curl} e-Y+Y, \operatorname{curl} e_{t}\right\rangle_{\Omega}(t) \\
&+2\left\langle\mu^{-1} \operatorname{curl} e, \operatorname{curl}\left(\tilde{E}_{t}-\partial_{t} \tilde{E}\right)\right\rangle_{\Omega}(t) \\
&=2\left\langle\hat{F}-\operatorname{curl} Y-\varepsilon \partial_{t} \tilde{E}_{t}, e_{t}\right\rangle_{\Omega}(t)-2\left\langle\mathcal{G}(\tilde{E}, Y), \operatorname{curl} e_{t}\right\rangle_{\Omega}(t) \\
&+2\left\langle\operatorname{curl}\left(\tilde{E}_{t}-\partial_{t} \tilde{E}\right), \mu^{-1} \operatorname{curl} e\right\rangle_{\Omega}(t) \\
&=- 2\left\langle\hat{\mathcal{F}}\left(\tilde{E}_{t}, Y\right), e_{t}\right\rangle_{\Omega}(t)-2 \partial_{t}\langle\mathcal{G}(\tilde{E}, Y), \operatorname{curl} e\rangle_{\Omega}(t) \\
&+2\left\langle\mathcal{G}(\tilde{E}, Y), \operatorname{curl}\left(\tilde{E}_{t}-\partial_{t} \tilde{E}\right)\right\rangle_{\Omega}(t) \\
&+2\langle\underbrace{\mu^{-1} \operatorname{curl}\left(\tilde{E}_{t}-\partial_{t} \tilde{E}\right)+\partial_{t} \mathcal{G}(\tilde{E}, Y)}_{=\mu^{-1} \operatorname{curl} \tilde{E}_{t}-\partial_{t} Y}, \operatorname{curl} e\rangle_{\Omega}(t)
\end{aligned}
$$


Thus, by integration

$$
\begin{aligned}
n_{e_{t}, e, 1}(t)= & z\left(e, e_{t}, \tilde{E}, Y ; 0\right)+2\left\langle\mathcal{G}(\tilde{E}, Y), \operatorname{curl}\left(\tilde{E}_{t}-\partial_{t} \tilde{E}\right)\right\rangle_{\Omega_{t}} \\
& -2(\underbrace{\langle\mathcal{G}(\tilde{E}, Y), \operatorname{curl} e\rangle_{\Omega}(t)}_{=: S_{1}(t)}-\underbrace{\left\langle\mu^{-1} \operatorname{curl} \tilde{E}_{t}-\partial_{t} Y, \operatorname{curl} e\right\rangle_{\Omega_{t}}}_{=: S_{2}(t)} \\
& +\underbrace{\left\langle\hat{\mathcal{F}}\left(\tilde{E}_{t}, Y\right), e_{t}\right\rangle_{\Omega_{t}}}_{=: S_{3}(t)}) .
\end{aligned}
$$

If $\tilde{E}_{t}=\partial_{t} \tilde{E}$, then (4.6) coincides with (3.5). As before, we choose $\alpha:=1-\rho$ and $\beta:=\gamma \rho$ and estimate the scalar products $S_{\ell}$ as follows:

$$
\begin{aligned}
2\left|S_{1}(t)\right| \leq & \alpha(t)\|\operatorname{curl} e\|_{\mu^{-1}, \Omega}^{2}(t)+\alpha^{-1}(t)\|\mathcal{G}(\tilde{E}, Y)\|_{\mu, \Omega}^{2}(t), \\
2\left|S_{2}(t)\right| \leq & \int_{0}^{t} \beta(s)\|\operatorname{curl} e\|_{\mu^{-1}, \Omega}^{2}(s) d s \\
& +\int_{0}^{t} \beta^{-1}(s)\left\|\mu^{-1} \operatorname{curl} \tilde{E}_{t}-\partial_{t} Y\right\|_{\mu, \Omega}^{2}(s) d s \\
2\left|S_{3}(t)\right| \leq & \int_{0}^{t} \gamma(s)\left\|e_{t}\right\|_{\varepsilon, \Omega}^{2}(s) d s+\int_{0}^{t} \gamma^{-1}(s)\left\|\hat{\mathcal{F}}\left(\tilde{E}_{t}, Y\right)\right\|_{\varepsilon^{-1}, \Omega}^{2}(s) d s .
\end{aligned}
$$

Inserting (4.7) into (4.6) yields

$$
\begin{aligned}
n_{e_{t}, e, \rho}(t) & \leq \int_{0}^{t} \gamma(s) n_{e_{t}, e, \rho}(s) d s+f_{\rho, \gamma}\left(\tilde{E}, \tilde{E}_{t}, Y ; t\right), \\
N_{e_{t}, e, \rho, \gamma}^{\prime}(t) & \leq \gamma(t) N_{e_{t}, e, \rho, \gamma}(t)+\gamma(t) f_{\rho, \gamma}\left(\tilde{E}, \tilde{E}_{t}, Y ; t\right) .
\end{aligned}
$$

Finally, we apply Gronwall's inequalities, Lemma 1 and 2 of the appendix, and complete the proof.

Theorem 6. Let approximations $\tilde{E}, \tilde{E}_{t}$ and $Y$ as in Theorem 4 or Theorem 5 be given. Moreover, let all the functionals be defined with the variants using the nonnegative terms. Then, the following two statements are equivalent:

(i) $\tilde{E}(0)=E_{0}$ and $\tilde{E}_{t}=\partial_{t} \tilde{E}$ and $m_{\rho, \gamma}\left(\tilde{E}, \tilde{E}_{t}, Y ; \cdot\right)=0$.

(ii) $\tilde{E}=E$ and $\tilde{E}_{t}=\partial_{t} E$ and $\mu Y=\operatorname{curl} E$.

The proof follows from the fact that $e_{t}=0$ implies the relation

$$
e(t)=\int_{0}^{t}\left(\tilde{E}_{t}-\partial_{t} \tilde{E}\right)(s) d s+e(0)
$$

Theorem 6 has a clear meaning. It shows that if the approximations $\tilde{E}, \tilde{E}_{t}$ satisfy $\tilde{E}_{t}=\partial_{t} \tilde{E}$ and the first initial condition $\tilde{E}(0)=E_{0}$ exactly, then the functional $m_{\rho, \gamma}\left(\tilde{E}, \tilde{E}_{t}, Y ; \cdot\right)$ vanishes if and only if the approximation $\tilde{E}$ equals $E$ and $\mu Y$ equals curl $E$. The assertions of the latter theorem remain valid if we replace the functional $m_{\rho, \gamma}\left(\tilde{E}, \tilde{E}_{t}, Y\right)$ by $M_{\rho, \gamma}\left(\tilde{E}, \tilde{E}_{t}, Y ; \cdot\right), \hat{m}_{\rho, \gamma}\left(\tilde{E}, \tilde{E}_{t}, Y ; \cdot\right)$ or $\hat{M}_{\rho, \gamma}\left(\tilde{E}, \tilde{E}_{t}, Y ; \cdot\right)$. Furthermore, we get again new variational formulations for our problems. 


\section{Estimates for the approximation of the whole solution}

By (2.6) (or the basic equations (2.1), (2.2)) we also get estimates for the errors $h, h_{t}$ of the magnetic fields $H, \partial_{t} H$ and their approximations $\tilde{H}, \tilde{H}_{t}$. E.g., by adding $-\left(\tilde{E}_{t}, \tilde{H}_{t}\right)+\mathrm{i} C_{\Lambda}(\tilde{E}, \tilde{H})$ to $(2.6)$ we obtain

$$
\left(e_{t}, h_{t}\right)-\mathrm{i} C_{\Lambda}(e, h)=(\check{F}, \check{G}):=(F, G)-\left(\tilde{E}_{t}, \tilde{H}_{t}\right)+\mathrm{i} C_{\Lambda}(\tilde{E}, \tilde{H}),
$$

which reads explicitly

$$
\begin{aligned}
& e_{t}-\varepsilon^{-1} \operatorname{curl} h=\check{F}=F-\tilde{E}_{t}+\varepsilon^{-1} \operatorname{curl} \tilde{H}, \\
& h_{t}+\mu^{-1} \operatorname{curl} e=\check{G}=G-\tilde{H}_{t}-\mu^{-1} \operatorname{curl} \tilde{E} \text {. }
\end{aligned}
$$

Therefore, we can estimate

$$
\begin{aligned}
\hat{n}_{\rho}\left[h_{t}, h\right](t) & :=\rho(t)\left\|h_{t}\right\|_{\mu, \Omega}^{2}(t)+\|\operatorname{curl} h\|_{\varepsilon^{-1}, \Omega}^{2}(t) \\
& \leq 2 n_{e_{t}, e, \rho}(t)+2\|\check{F}\|_{\varepsilon, \Omega}^{2}(t)+2\|\check{G}\|_{\mu, \Omega}^{2}(t) \\
& \leq 2 \inf _{Y, \gamma} m_{\rho, \gamma}\left(\tilde{E}, \tilde{E}_{t}, Y ; t\right)+2\|\check{F}\|_{\varepsilon, \Omega}^{2}(t)+2\|\check{G}\|_{\mu, \Omega}^{2}(t),
\end{aligned}
$$

which yields

$$
\begin{aligned}
& n_{e_{t}, e, \rho}(t)+\hat{n}_{\rho}\left[h_{t}, h\right](t) \\
& =\left\|e_{t}\right\|_{\varepsilon, \Omega}^{2}(t)+\rho(t)\|\operatorname{curl} e\|_{\mu^{-1}, \Omega}^{2}(t)+\rho(t)\left\|h_{t}\right\|_{\mu, \Omega}^{2}(t)+\|\operatorname{curl} h\|_{\varepsilon^{-1}, \Omega}^{2}(t) \\
& \leq 3 \inf _{Y, \gamma} m_{\rho, \gamma}\left(\tilde{E}, \tilde{E}_{t}, Y ; t\right)+2\|\check{F}\|_{\varepsilon, \Omega}^{2}(t)+2\|\check{G}\|_{\mu, \Omega}^{2}(t) .
\end{aligned}
$$

Of course, similar estimates hold for the other norms and functionals and the estimates simplify in an obvious way if $\rho$ or $\gamma$ are positive constants. The vector fields $(\check{F}, \check{G})$ measure the error in the original first order equation (2.6). Moreover, $\left(e_{t}, h_{t}\right)$ may be replaced by $\partial_{t}(e, h)$ if for the approximations sufficient regularity is available. In this case, the error in the first order equation is

$$
(\check{F}, \check{G})=(F, G)-\left(\partial_{t}-\mathrm{i} C_{\Lambda}\right)(\tilde{E}, \tilde{H})=\left(\partial_{t}-\mathrm{i} C_{\Lambda}\right)(e, h) .
$$

\section{Appendix A. Gronwall inequalities}

Gronwall's inequalities (in the differential and integral forms) are widely used in the theory of ordinary differential equations. For the convenience of the reader, we present below two forms of these estimates, which are convenient for our analysis. Since these estimates slightly differ from commonly known forms (as, e.g., in [2]), we supply two lemmas.

Lemma 1. (Differential form) Let $u \in C_{p}^{1}(\bar{I}):=C_{p}^{1}(\bar{I}, \mathbf{R})$ and $\varphi, \psi \in C^{0}(\bar{I}):=$ $C^{0}(\bar{I}, \mathbf{R})$ with $\varphi \geq 0$. If the inequality

$$
u^{\prime} \leq \varphi u+\psi
$$

holds in $I$, then

$$
u(t) \leq \exp (\Phi(t))\left(u(0)+\int_{0}^{t} \exp (-\Phi(s)) \psi(s) d s\right), \quad \Phi(t):=\int_{0}^{t} \varphi(s) d s,
$$

holds for all $t \in \bar{I}$. 
If $\varphi$ is a nonnegative constant, then for all $t \in \bar{I}$

$$
u(t) \leq \exp (\varphi t)\left(u(0)+\int_{0}^{t} \exp (-\varphi s) \psi(s) d s\right) .
$$

If $\psi \leq c \in \mathbf{R}$, then for all $t \in \bar{I}$ we have

$$
u(t) \leq(u(0)+c t) \exp (\Phi(t)) .
$$

Lemma 2. (Integral form) Let $u, \varphi, \psi \in C^{0}(\bar{I})$ with $\varphi \geq 0$. If for all $t \in \bar{I}$

$$
u(t) \leq \int_{0}^{t} \varphi(s) u(s) d s+\psi(t)
$$

then for all $t \in \bar{I}$

$$
u(t) \leq \exp (\Phi(t)) \int_{0}^{t} \exp (-\Phi(s)) \varphi(s) \psi(s) d s+\psi(t) .
$$

If $\varphi$ is a nonnegative constant, then for all $t \in \bar{I}$

$$
u(t) \leq \varphi \exp (\varphi t) \int_{0}^{t} \exp (-\varphi s) \psi(s) d s+\psi(t) .
$$

If $\psi \leq c \in \mathbf{R}$, then for all $t \in \bar{I}$

$$
u(t) \leq c \exp (\Phi(t))
$$

Acknowledgements. The authors thank the University of Jyväskylä for supporting this research.

\section{References}

[1] Bildhauer, M., M. Fuchs, and S. Repin: Duality based a posteriori error estimates for higher order variational inequalities with power growth functionals. - Ann. Acad. Sci. Fenn. Math. 33, 2008, 475-490.

[2] Evans, L.: Partial differential equations. - Amer. Math. Soc., Providence, Rhode Island, 2003.

[3] FuCHS, M., and S. REPIN: Estimates for the deviation from the exact solutions of variational problems modeling certain classes of generalized Newtonian fluids. - Math. Methods Appl. Sci. 29, 2006, 2225-2244.

[4] Fuchs, M., and S. Repin: Estimates of the deviations from the exact solutions for variational inequalities describing the stationary flow of certain viscous incompressible fluids. - Math. Methods Appl. Sci. 33, 2010, 1136-1147.

[5] Kuhn, P., and D. PAuly: Regularity results for generalized electro-magnetic problems. Analysis (Munich) 30, 2010, 225-252.

[6] Ladyzhenskaya, O. A.: The boundary value problems of mathematical physics. - Springer, New York, 1985.

[7] LEIS, R.: Initial boundary value problems in mathematical physics. - Teubner, Stuttgart, 1986.

[8] NeittaAnmäKi, P., and S. Repin: Reliable methods for computer simulation. Error control and a posteriori estimates. - Elsevier, Amsterdam, 2004.

[9] PAuly, D.: Low frequency asymptotics for time-harmonic generalized Maxwell equations in nonsmooth exterior domains. - Adv. Math. Sci. Appl. 16:2, 2006, 591-622.

[10] Pauly, D.: Generalized electro-magneto statics in nonsmooth exterior domains. - Analysis (Munich) 27:4, 2007, 425-464. 
[11] PAuly, D.: Hodge-Helmholtz decompositions of weighted Sobolev spaces in irregular exterior domains with inhomogeneous and anisotropic media. - Math. Methods Appl. Sci. 31, 2008, 1509-1543.

[12] Pauly, D.: Complete low frequency asymptotics for time-harmonic generalized Maxwell equations in nonsmooth exterior domains. - Asymptot. Anal. 60:3-4, 2008, 125-184.

[13] Pauly, D., and S. RePin: Functional a posteriori error estimates for elliptic problems in exterior domains. - J. Math. Sci. (N. Y.) 162:3, 2009, 393-406.

[14] Pauly, D., and S. RePin: Two-sided a posteriori error bounds for electro-magneto static problems. - J. Math. Sci. (N. Y.) 166:1, 2010, 53-62.

[15] Pauly, D., and T. Rossi: Computation of generalized time-periodic waves using differential forms, exact controllability, least-squares formulation, conjugate gradient method and discrete exterior calculus: Part I. Theoretical considerations. - Reports of the Dept. of Mathematical Information Technology, Univ. of Jyväskylä, Series B. Scientific Computing, No. B. 16, 2008.

[16] Repin, S.: A posteriori error estimation for nonlinear variational problems by duality theory. - Zap. Nauchn. Sem. S.-Peterburg. Otdel. Mat. Inst. Steklov. (POMI) 243, 1997, 201-214.

[17] Repin, S.: A posteriori error estimation for variational problems with uniformly convex functionals. - Math. Comp. 69:230, 2000, 481-500.

[18] Repin, S.: Two-sided estimates of deviation from exact solutions of uniformly elliptic equations. - Proc. St. Petersburg Math. Society IX, 2001, 143-171.

[19] Repin, S.: Estimates of deviation from exact solutions of initial-boundary value problems for the heat equation. - Atti Accad. Naz. Lincei Cl. Sci. Fis. Mat. Natur. Rend. Lincei (9) Mat. Appl. 13, 2002, 121-133.

[20] Repin, S.: Estimates of deviations from exact solutions of elliptic variational inequalities. Zap. Nauchn. Sem. S.-Peterburg. Otdel. Mat. Inst. Steklov. (POMI) 326, 2007, 147-164.

[21] Repin, S.: A posteriori error estimates for partial differential equations. - Walter deGruyter, Berlin, 2008.

[22] RePin, S.: Estimates of deviations from exact solutions of initial boundary value problem for the wave equation. - J. Math. Sci. (N. Y.) 159:2, 2009, 229-240.

Received 14 January 2011 